\title{
GAUGE INVARIANT FUNCTIONS OF CONNECTIONS
}

\author{
AMBAR SENGUPTA
}

(Communicated by Ronald Stern)

\begin{abstract}
It is shown that, for certain gauge groups, central functions of the holonomy variables do not determine connections up to gauge equivalence. It is also shown that, for a large class of compact groups, such functions do determine connections up to gauge equivalence. It is then shown that, for the latter type of gauge groups, the Euclidean quantum gauge field measure is determined by the expectation values of the Wilson loop variables (products of characters evaluated on holonomies).
\end{abstract}

\section{INTRODUCTION}

In this paper we show $(\S 3)$ that, for certain noncompact gauge groups, gauge invariant functions of the holonomy variables do not determine gauge fields (i.e., connections on principal bundles) up to gauge equivalence. In contrast, we show (Theorem 1) that, for a wide class of compact gauge groups, such functions do determine gauge fields up to gauge equivalence. As a consequence we prove (Theorem 4) that for such groups the Yang-Mills functional integral measure is determined by the Wilson loop expectation values (these are expectation values of products of characters evaluated on holonomy variables). In Theorem 2 we answer the following question affirmatively for certain classes of classical compact groups: Let $\left\{x_{i}\right\}_{i \in I},\left\{x_{i}^{\prime}\right\}_{i \in I}$ be families of elements in a group $G$ such that $x_{i_{1}} \cdots x_{i_{k}}$ is conjugate to $x_{i_{1}}^{\prime} \cdots x_{i_{k}}^{\prime}$ for every $k \geq 1$ and every $i_{1}, \ldots, i_{k} \in$ $I$; then is there a $g \in G$ such that $g x_{i} g^{-1}=x_{i}^{\prime}$ for all $i \in I$ ?

The Wilson loop variables are often taken as the fundamental objects describing gauge-equivalence classes of gauge fields especially in the context of quantum gauge theories (see [Sei, Fr]). The problem of reconstructing gauge fields from Wilson loop variables was discussed in an informal way in [Gi]. Kobayashi [Ko] announced a description of gauge-equivalence classes of connections in terms of holonomies. This approach has been investigated and clarified in a more complete manner by Driver [Dr].

\section{THE PROBLEM}

Mathematically, the problem may be formulated as follows: Let $\pi: P \rightarrow M$ be a smooth principal bundle over a connected manifold $M$ with gauge group

Received by the editors October 26, 1992.

1991 Mathematics Subject Classification. Primary 81T13; Secondary 81T08.

Key words and phrases. Gauge invariance, Wilson loops, quantum gauge field. 
(= structure group) a Lie group $G$. For a connection $\omega$ on $P$ and a piecewise smooth closed loop $C$ in $M$ based at some point $m \in M$ we denote by $g_{u}(C ; \omega)$ the holonomy around $C$ starting at a point $u$ on the fiber $\pi^{-1}(m)$ over $m$. By a gauge transformation $\phi$ on $P$ we mean a diffeomorphism $\phi: P \rightarrow P$ for which

(i) $\phi(p g)=\phi(p) g$ for every $p \in P$ and $g \in G$ and

(ii) $\phi$ maps each fiber $\pi^{-1}(x)$ onto itself for every $x \in M$.

The question then is: if $\omega$ and $\omega^{\prime}$ are connections on $P$ such that $g_{u}(C ; \omega)$ and $g_{u}\left(C ; \omega^{\prime}\right)$ are conjugate elements in $G$ for every piecewise smooth closed loop $C$ based at $m$, then is there a gauge transformation $\phi$ of $P$ for which $\phi^{*} \omega=\omega^{\prime}$ ?

In most formulations one looks in fact at the Wilson loop variable $\chi\left(g_{u}(C ; \omega)\right)$, where $\chi$ is an arbitrary of $G$. Of course, if $g, g^{\prime} \in G$ are conjugate in $G$ then $\chi(g)=\chi\left(g^{\prime}\right)$, while the converse holds when $G$ is compact. In the physical literature, an affirmative answer to the above question is generally assumed.

\section{Counterexamples}

Let $G$ be the connected component of the identity in the Poincare group. An element of $G$ is of the form $(a, \Lambda)$, where $a \in R^{4}$ and $\Lambda$ is a proper orthochronous Lorentz transformation (i.e., $\Lambda=\left(\Lambda_{i j}\right)_{0 \leq i, j \leq 3}$ is a $4 \times 4$ unitdeterminant real matrix with $\Lambda_{00}>0$ and for which $\Lambda^{T} F \Lambda=F$, where $\Lambda^{T}$ denotes the transpose of $\Lambda$ and $F$ is the $4 \times 4$ diagonal matrix whose $(0,0)$ th entry is +1 while all the other diagonal entries are -1$)$. The law of composition is defined by

$$
(a, g)(b, h)=(a+g b, g h)
$$

for $(a, g),(b, h) \in G$. For notational purposes, an element $v$ of $R^{4}$ will be taken as a column vector with components $v_{i}$ indexed by $i=0,1,2,3$.

Let $H$ denote the subgroup of $G$ consisting of translations, i.e., $H=$ $\left\{(a, I): a \in R^{4}\right\}$, where $I$ is the $4 \times 4$ identity matrix.

The map $\Phi: H \rightarrow H:(a, I) \mapsto(F a, I)$ is an isomorphism ( $F$ is the matrix mentioned above). Note that $(F a)^{T} F(F a)=a^{T} F a$ and that the 0th component of $F a$ is equal to the 0th component of $a$.

Now if $(a, I),(x, y) \in G$ then $(x, y)(a, I)(x, y)^{-1}=(y a, I)$. A basic property of the group of proper orthochronous Lorentz transformations is that for any $a, b \in R^{4}$, with $a^{T} F a=b^{T} F b$ and $a_{0}$ and $b_{0}$ of the same sign, there exists a proper orthochronous Lorentz matrix $y$ with $y a=b$ (see, e.g., [Va]).

Therefore, we conclude that if $(a, I),(b, I) \in G$ with $a^{T} F a=b^{T} F b$ and if $a_{0} b_{0}>0$, then there is a $\gamma \in G$ with $\gamma(a, I) \gamma^{-1}=(b, I)$. In particular, this is true of $b=F a$.

However, it is also clear that there is no $\gamma \in G$ for which $\gamma\left(e_{i}, I\right) \gamma^{-1}=$ $\left(F e_{i}, I\right)$ holds for every element $e_{i}$ of a basis $\left(e_{0}, e_{1}, e_{2}, e_{3}\right)$ of $R^{4}$, for that would imply that $\gamma=(x, F)$ for some $x \in R^{4}$, and this would not be possible since $(x, F)$ is not an element of $G$.

Let $\pi: P=R^{4} \times G \rightarrow R^{4}$ be the trivial principal bundle over four-dimensional Euclidean space $R^{4}$. We may view $P$ as the bundle of 'affine (proper orthochronous) Lorentz frames' over $R^{4}$. Fix a point $m \in R^{4}$. 
Let $s$ denote the section $s: R^{4} \rightarrow P: x \mapsto s(x)=(x,(0, I))$ of the bundle $P$. Write $u=s(m)$.

Choose smooth 1-forms $a_{0}, a_{1}, a_{2}, a_{3}$ on $R^{4}$ and smooth loops $C_{0}, C_{1}$, $C_{2}, C_{3}$, all based at $m$, such that $\int_{C_{j}} a_{i}=\delta_{i j}$ (where $\delta_{i j}$ is 0 if $i \neq j$ and is 1 if $i=j$ ). For instance, we can take each $a_{i}$ to be of the form $x_{j} d x_{k} / \pi$ and each $C_{i}$ a unit circle parallel to the $\left(x_{j}-x_{k}\right)$-plane. Now define the $R^{4}$-valued 1-form $A$ by

$$
A=a_{0} e_{0}+a_{1} e_{1}+a_{2} e_{2}+a_{3} e_{3} \text {, }
$$

where $\left(e_{0}, e_{1}, e_{2}, e_{3}\right)$ is the standard basis of $R^{4}$.

Let $\omega$ denote the connection on $P$ specified by requiring that $\omega\left(s_{*}(X)\right)=$ $(A(X), 0)$ for every vector $X$ tangent to $R^{4}$. If $C$ is any piecewise smooth closed loop in $R^{4}$ based at $m$ then $g_{u}(C ; \omega)=\left(\int_{C} A, I\right)$. Define a connection $\omega^{\prime}$ on $P$ in exactly the same way except with $A$ replaced by $F \circ A$ in the definition of $\omega$. So if $g_{u}(C ; \omega)=(a, I)$ then $g_{u}\left(C ; \omega^{\prime}\right)=(F a, I)$. Thus $\omega$ and $\omega^{\prime}$ may te viewed as 'generalized affine connections' (see [KN, §3.3]) on $R^{4}$.

Therefore, by our previous remarks, it follows that

(i) for every piecewise smooth loop $C$, based at $m$, there exists a $\gamma \in G$ with $\gamma^{-1} g_{u}(C ; \omega) \gamma=g_{u}\left(C ; \omega^{\prime}\right)$, and

(ii) there is no $\gamma \in G$ for which $\gamma^{-1} g_{u}(C ; \omega) \gamma=g_{u}\left(C ; \omega^{\prime}\right)$ holds for every piecewise smooth loop $C$.

Therefore, there is no gauge transformation $\psi$ of $P$ for which $\psi^{*} \omega=\omega^{\prime}$. For if such a $\psi$ were to exist then there would be a $\gamma \in G$ defined by $\psi(u)=$ $u \gamma$, and then one would have

$$
g_{u}\left(C ; \omega^{\prime}\right)=\gamma^{-1} g_{u}(C ; \omega) \gamma
$$

for all piecewise smooth closed loops $C$ based at $m$.

Variants of the above example include the group of all proper Euclidean motions in $R^{n}(n>1)$ and certain finite groups obtained as semidirect products (or see [Bu2]). Moreover, the product of such a finite group with any compact Lie group (dim $\geq 1)$ yields another example-this time with a compact Lie group of dimension $\geq 1$. Finite groups and their products with other groups are relevant only for lattice gauge theories and continuum theories on nonsimply connected base spaces. An example can be manufactured along the above lines with $G$ as the semidirect product of $R^{4}$ and $\mathrm{SU}(2)$, and this can be used in the context of principal bundles over arbitrary base manifolds of dimension at least 1 .

These examples are related to the problem of finding groups which have noninner automorphisms which preserve each conjugacy class. The question as to whether such groups exist was raised first by Burnside in his book [Bu1]-he later constructed such examples involving finite groups [Bu2]. The results of the following section show that for many of the classical compact groups any conjugacy class-preserving automorphism must be inner.

\section{ORTHOGONAL AND UNITARY GROUPS}

Theorem 1. Let $G$ be a compact Lie group for which the conclusion Theorem 2 holds (see the statement in Theorem 2 for a list of examples). Let $\pi: P \rightarrow M$ be a principal G-bundle over a connected manifold $M$. Fix an $m \in M$ and any 
point $u$ on the fiber $\pi^{-1}(m)$ over $m$. Suppose $\omega$ and $\omega^{\prime}$ are connections on $P$ such that $\chi\left(g_{u}(C ; \omega)\right)=\chi\left(g_{u}\left(C ; \omega^{\prime}\right)\right)$ for every piecewise smooth closed loop in $M$ based at $m$ and every character $\chi$. (The holonomy around $C$, starting at $u$, with respect to the connection $\omega$ is denoted $g_{u}(C ; \omega)$.) Then there is a gauge transformation $\phi: P \rightarrow P$ such that $\phi^{*} \omega=\omega^{\prime}$.

Proof. Since characters of $G$ separate conjugacy classes, each $g_{u}(C ; \omega)$ is conjugate to $g_{u}\left(C ; \omega^{\prime}\right)$. In particular, the product of any finite number of $g_{u}(C ; \omega)$ 's, being of the form $g_{u}\left(C_{n} \cdots C_{1} ; \omega\right)$ with $C_{n} \cdots C_{1}$ the composite of piecewise smooth loops $C_{i}$ based at $m$, is conjugate to the corresponding product of the $g_{u}\left(C ; \omega^{\prime}\right)$ 's. (Of course, $C$ refers to piecewise smooth closed loops based at $m$.) Then by Theorem 2 there is a $\gamma \in G$ such that $\gamma g_{u}(C ; \omega) \gamma^{-1}=g_{u}\left(C ; \omega^{\prime}\right)$ for all $C$. Using a local cross-section of $\pi: P \rightarrow M$ in a neighborhood of $m$ it is possible to construct a gauge transformation $\xi: P \rightarrow P$ such that $\xi(u)=u \gamma^{-1}$ (here $u$ is the fixed point on $\pi^{-1}(m)$ chosen earlier).

Then

$$
g_{u}\left(C ; \xi^{*} \omega\right)=\gamma g_{u}(C ; \omega) \gamma^{-1}=g_{u}\left(C ; \omega^{\prime}\right) .
$$

Now define a gauge transformation $\psi: P \rightarrow P$ as follows. For $p \in P$ define $\psi(p)$ to be the point on the fiber through $p$ which is obtained by parallel translating $p$ according to $\xi^{*} \omega$ along a piecewise smooth path from $\pi(p)$ to $m$ and then parallel translating according to $\omega^{\prime}$ back along the same path (in reverse direction) from $m$ to $\pi(p)$. That $\psi$ is well defined follows from $(*)$. That $\psi$ is smooth may be seen by taking local cross-sections. Then $\psi^{*}\left(\xi^{*} \omega\right)=$ $\omega^{\prime}$, as may be readily verified. Thus $\phi=\xi \circ \psi$ is a gauge transformation for which $\phi^{*} \omega=\omega^{\prime}$.

The group $G$ acts on $G^{m}$ by $\left(g,\left(x_{1}, \ldots, x_{m}\right)\right)=\left(g x_{1} g^{-1}, \ldots, g x_{m} g^{-1}\right)$, and we denote the quotient by $G^{m} / G$. A function $f$ on $G^{m}$ will be called $G$-invariant if

$$
f\left(g x_{1} g^{-1}, \ldots, g x_{m} g^{-1}\right)=f\left(x_{1}, \ldots, x_{m}\right)
$$

for every $g, x_{1}, \ldots, x_{m} \in G$.

Theorem 2. Let $G$ be a product of groups from the following list: abelian groups, the unitary groups $\mathrm{U}(n)$, special unitary groups $\mathrm{SU}(n)$, orthogonal groups $\mathrm{O}(n)$, and the odd special orthogonal groups $\mathrm{SO}(2 n+1)$. Suppose that $\left\{g_{\alpha}\right\}_{\alpha \in I}$ and $\left\{g_{\alpha}^{\prime}\right\}_{\alpha \in I}$ are families of elements of $G$ such that for every $\alpha_{1}, \ldots, \alpha_{k} \in I$ the product $g_{\alpha_{1}} \cdots g_{\alpha_{k}}$ is conjugate in $G$ to $g_{\alpha_{1}}^{\prime} \cdots g_{\alpha_{k}}^{\prime}$. Then there is an element $\gamma \in G$ such that $g_{\alpha}^{\prime}=\gamma g_{\alpha} \gamma^{-1}$ for every $\alpha \in I$.

Proof. If $G$ is abelian then the result is trivial. Moreover, if the result holds for each member of a family of groups then it does for their product. Therefore, it will suffice to consider the cases where $G$ is one of the groups $\mathrm{U}(n), \mathrm{SU}(n)$, $\mathrm{O}(n)$, or $\mathrm{SO}(2 n+1)$. Henceforth we shall assume that $G$ is one of these matrix groups. Since each of these groups is compact, it may be seen that it suffices to assume that the indexing set is finite. We will take $I=\{1, \ldots, m\}$.

The hypothesis implies that the trace of the product $g_{i_{1}} \cdots g_{i_{k}}$ equals the trace of $g_{i_{1}}^{\prime} \cdots g_{i_{k}}^{\prime}$ for every positive integer $k$ and every $i_{1}, \ldots, i_{k} \in\{1, \ldots, m\}$. It is only this apparently weaker hypothesis that will be used. Consider any 
$g \in G$. By considering the diagonalized form of the matrix $g$ we see that there is a polynomial $P(X)$ such that $P(g)=g^{-1}$. If $g^{\prime} \in G$ is conjugate to $g$ then $P\left(g^{\prime}\right)=g^{\prime-1}$. Therefore, we have

$$
\operatorname{Tr}\left(g_{i_{1}}^{\varepsilon_{1}} \cdots g_{i_{k}}^{\varepsilon_{k}}\right)=\operatorname{Tr}\left(g_{i_{1}}^{\varepsilon_{1}} \cdots g_{i_{k}}^{\prime} \varepsilon_{k}\right)
$$

for every $k \geq 1, i_{1}, \ldots, i_{k} \in\{1, \ldots, m\}$, and $\varepsilon_{1}, \ldots, \varepsilon_{i} \in\{1,-1\}$.

We start with the case $G=\mathrm{U}(n)$. Since $G^{m} / G$ is compact Hausdorff, the Stone-Weierstrass theorem tells us that it will suffice to show $f\left(g_{1}, \ldots, g_{m}\right)=$ $f\left(g_{1}^{\prime}, \ldots, g_{m}^{\prime}\right)$ for every $G$-invariant continuous function $f: G^{m} \rightarrow C$. Again by the Stone-Weierstrass theorem we can approximate $f$ arbitrarily closely by functions $P$ on $G^{m}$ for which $P\left(g_{1}, \ldots, g_{m}\right)$ are polynomials in the entries of the matrices $g_{i}$ and their conjugates. Replacing such a $P$ by its average over $G$ under the adjoint action (i.e., by

$$
\left(x_{1}, \ldots, x_{m}\right) \mapsto \int_{G} P\left(g x_{1} g^{-1}, \ldots, g x_{m} g^{-1}\right) d g,
$$

where $d g$ is the Haar measure of unit mass on $G$ ) we may and will assume that $P$ is $G$-invariant. It will suffice to show that $P\left(g_{1}, \ldots, g_{m}\right)=P\left(g_{1}^{\prime}, \ldots, g_{m}^{\prime}\right)$. Note that $P\left(X_{1}, \ldots, X_{m}\right)$ is still a polynomial in the matrix entries of the $X_{i}$ 's and their conjugates and is defined for all $n \times n$ complex matrices, not just unitary ones; moreover, $P$ is $G$-invariant in the sense that $P\left(X_{1}, \ldots, X_{m}\right)$ has the same value if each $X_{i}$ is replaced by $g X_{i} g^{-1}$ with the same $g \in$ $G$. By its polynomial nature, $P$ is a linear combination of terms of the form $Q\left(X_{i_{1}} \otimes \cdots \otimes X_{i_{k}} \otimes X_{j_{1}}^{*} \otimes \cdots \otimes X_{j_{i}}^{*}\right)$, where $Q \in\left(\operatorname{End}_{C}\left(\otimes^{k+l} C^{n}\right)\right)^{*}$ has the form

$$
Q(B)=\left\langle B\left(e_{\beta_{1}} \otimes \cdots \otimes e_{\delta_{l}}\right), e_{\alpha_{1}} \otimes \cdots \otimes e_{\theta_{l}}\right\rangle
$$

with $\left(e_{1}, \ldots, e_{n}\right)$ being the standard basis of $C^{n}$ and $\langle\cdot, \cdot\rangle$ the usual inner product on $C^{n}$. By linear algebra there exists $Q^{\prime} \in \operatorname{End}_{C}\left(\otimes^{k+l} C^{n}\right)$ such that $Q(B)=\operatorname{Tr}\left(B Q^{\prime}\right)$ for all $B \in \operatorname{End}_{C}\left(\otimes^{k+l} C^{n}\right)$. Since $P$ is $G$-invariant, we may and will, by averaging as before if necessary, assume that each $Q^{\prime}$ commutes with $g \otimes \cdots \otimes g=\otimes^{k+l} g$ for every $g \in G$. Therefore, by a classical result (see [We]), $Q^{\prime}$ is a linear combination of the permutation operators $P_{\sigma}\left(\sigma \in S_{k+l}\right)$

$$
P_{\sigma}\left(v_{1} \otimes \cdots v_{k+l}\right)=v_{\sigma_{1}} \otimes \cdots \otimes v_{\sigma_{k+l}} .
$$

Now if $\sigma=C_{1} \cdots C_{s}$ is the cycle decomposition of $\sigma$ then it may be verified that (see [GKS, Lemma 3.5])

$$
\operatorname{Tr}\left(\left(A_{1} \otimes \cdots \otimes A_{k+l}\right) P_{\sigma}\right)=\prod_{i=1}^{s} \operatorname{Tr}\left(A^{C_{i}}\right),
$$

where $A^{C_{i}}$ is the product $A_{\rho_{1}} \cdots A_{\rho_{f}}$ if $C_{i}$ is the cycle $\left(\rho_{1} \cdots \rho_{f}\right)$. Relation (2) and the fact that $g^{*}=g^{-1}$ for $g \in G=\mathrm{U}(n)$ now imply that $Q\left(g_{i_{1}} \otimes \cdots \otimes g_{i_{k}} \otimes g_{j_{1}}^{*} \otimes \cdots \otimes g_{j_{i}}^{*}\right)$ equals the corresponding quantity with the $g_{i}$ 's replaced by the $g_{i}^{\prime}$ 's. By taking linear combinations, we obtain $P\left(g_{1}, \ldots, g_{m}\right)=$ $P\left(g_{1}^{\prime}, \ldots, g_{m}^{\prime}\right)$ as desired, and we are done with the case $\mathrm{U}(n)$.

The case $G=\mathrm{SU}(n)$ is an immediate consequence of the $\mathrm{U}(n)$ case. This is obtained by scaling the $\gamma$ (see the statement of the theorem for notation) obtained by the $\mathrm{U}(n)$ argument by a suitable factor to ensure that it lies in $\mathrm{SU}(n)$. 
We turn to the case $G=\mathrm{O}(n)$. The $\mathrm{U}(n)$ case implies (with notation as usual) that there is a $\gamma \in U(n)$ such that $\gamma g_{i} \gamma^{-1}=g_{i}^{\prime}$ for all $i=1, \ldots, m$. Here we are looking at $R^{n} \subset C^{n}$ and $\mathrm{O}(n) \subset \mathrm{U}(n)$. Let $H$ be the closed subgroup generated by the $g_{i}$ 's. Write $x^{\prime}$ for $\gamma x \gamma^{-1}$. Since $\operatorname{Tr}(x)=\operatorname{Tr}\left(x^{\prime}\right)$, the (real) characters of the two representations $H \rightarrow \mathrm{O}(n): x \mapsto x$ and $H \rightarrow$ $\mathrm{O}(n): x \mapsto x^{\prime}$ are equal, and therefore these two real representations decompose into the same sequence of irreducible (real) representations and hence are orthogonally equivalent. That is, there is a $\tilde{g} \in \mathrm{O}(n)$ with $\tilde{g} h \tilde{g}^{-1}=h^{\prime}$ for all $h \in H$. In particular, this holds for $h$ equal to any $g_{i}$. This takes care of the $\mathrm{O}(n)$ case. For $n$ odd, the result for $G=\mathrm{SO}(n)$ follows from the result for $\mathrm{O}(n)$ as the $\mathrm{SU}(n)$ case followed from $\mathrm{U}(n)$.

Remark. The results above also hold for the symplectic groups. In the proof of Theorem 2 we saw that the case $\mathrm{SO}(2 n+1)$ followed immediately from the case of the orthogonal groups. That argument does not go through for the groups $\mathrm{SO}(2 n)(n>1)$. For $\mathrm{SO}(4)$ we have the following result.

Proposition 3. Let $G$ be a compact group with the property $(\mathrm{P})$ that:

(P) If $\left\{x_{i}\right\}_{i \in I}$ and $\left\{x_{i}^{\prime}\right\}_{i \in I}$ are families of elements in the group such that $x_{i_{1}} \cdots x_{i_{k}}$ is conjugate to $x_{i_{1}}^{\prime} \cdots x_{i_{k}}^{\prime}$, for every positive integer $k$ and every $i_{1}, \ldots$, $i_{k} \in I$, and if $\left\{x_{i}\right\}_{i \in I}$ generates a subgroup whose closure is connected, then there exists an element $g$ in the group for which $g x_{i} g^{-1}=x_{i}^{\prime}$ for all $i \in I$.

Suppose $G^{\prime}$ is a compact group having a finite normal subgroup $D$ such that $G^{\prime} / D$ is isomorphic to $G$. Then $G^{\prime}$ also has the property $(\mathrm{P})$. (P).

The groups $\operatorname{Spin}(2 n+1), \operatorname{Pin}(n), \mathrm{SO}(4)$, and $\operatorname{Spin}(4)$ have the property

Remark. A glance at the proof of Theorem 1 shows that the theorem holds for any compact Lie group $G$ having property $(\mathrm{P})$ provided that the holonomy group involved (of $\omega$ in the notation of Theorem 1) is connected-a condition which is always satisfied if the base manifold is simply connected.

Proof of Proposition 3. Identify $G$ with $G^{\prime} / D$, and let $p: G^{\prime} \rightarrow G$ be the quotient map. Let $\left\{x_{i}\right\}_{i \in I}$ and $\left\{x_{i}^{\prime}\right\}_{i \in I}$ be families of elements of $G^{\prime}$ such that $x_{i_{1}} \cdots x_{i_{k}}$ is conjugate to $x_{i_{1}}^{\prime} \cdots x_{i_{k}}^{\prime}$, for every positive integer $k$ and every $i_{1}, \ldots, i_{k} \in I$, and suppose $\left\{x_{i}\right\}_{i \in I}$ generates a subgroup $H$ of $G^{\prime}$ whose closure is connected. It is readily seen using $(\mathrm{P})$ that the assignment $x_{i} \mapsto x_{i}^{\prime}$ sets up a well-defined homomorphism $f: H \rightarrow G^{\prime}$ and that $f(h)$ is conjugate to $h$ for every $h \in H$. Since $G^{\prime}$ is compact, any neighborhood $U$ of the identity $e \in G^{\prime}$ contains a neighborhood $V$ of $e$ for which $g_{V g^{-1}}=V$ for all $g \in G^{\prime}$. Then $f^{-1}(V) \subset V$ because $f(h)$ is conjugate to $h$ for all $h \in H$. Thus $f$ is continuous and extends to a continuous homomorphism, also denoted by $f$, on the closure $\bar{H}$. We still have $f(h)$ conjugate to $h$ for all $h \in \bar{H}$. The property (P) for $G$ now implies that there is a $g \in G$ such that $p(f(h))=g p(h) g^{-1}$ for all $h \in \bar{H}$. Picking a $g^{\prime} \in p^{-1}(\{g\})$ we then have $p(f(h))=p\left(g^{\prime} h g^{-1}\right)$ for all $h \in \bar{H}$. So $f(h)\left(g^{\prime} h g^{-1}\right)^{-1} \in D$ for all $h \in \bar{H}$. Therefore, since $f$ is continuous, and $\bar{H}$ connected, and $D$ discrete, we conclude that $f(h)=g^{\prime} h g^{\prime-1}$ for all $h \in \bar{H}$. In particular, taking $h=x_{i}, x_{i}^{\prime}=g^{\prime} x_{i} g^{\prime-1}$ for all $i \in I$.

The second part follows from the first part, the facts that $\operatorname{Spin}(k) /\{1,-1\}$ is isomorphic to $\mathrm{SO}(k), \operatorname{Pin}(n) /\{1,-1\}$ is isomorphic to $\mathrm{O}(n)$, and $\mathrm{SO}(4) /$ 
$\{1,-1\}$ is isomorphic to $\mathrm{SO}(3) \times \mathrm{SO}(3)$, and the property $(\mathrm{P})$ for $\mathrm{SO}(2 n+1)$ which was proved in Theorem 2 .

\section{Specification of the Yang-Mills measure BY WILSON LOOP EXPECTATIONS}

We use the following notation: $\pi: P \rightarrow M$ is a principal $G$-bundle over the manifold $M, \mathscr{A}$ is the set of all connection forms on this bundle, $m$ is a fixed point in $M, u$ is a fixed point on the fiber $\pi^{-1}(m)$ over $m$, and $g_{u}(C ; \omega)$ denotes the holonomy of the connection $\omega$ around a closed loop in $M$ based at $m$ starting at $u$. Let $\mathscr{K}$ be a set of piecewise smooth closed loops in $M$, all based at some point $m \in M$. Assume that if $C_{1}, C_{2} \in \mathscr{K}$ then the composite loop $C_{1} \circ C_{2}$ also belongs to $\mathscr{K}$. In the application to continuum gauge theories $\mathscr{K}$ is large enough so that if $\omega, \omega^{\prime} \in \mathscr{A}$ are such that $g_{u}(K ; \omega)$ equals $g_{u}\left(K ; \omega^{\prime}\right)$ for every $K \in \mathscr{K}$ then $g_{u}(C ; \omega)$ equals $g_{u}\left(C ; \omega^{\prime}\right)$ for every piecewise smooth closed loop $C$ based at $m$. In the context of lattice gauge theories $\mathscr{K}$ would be the set of all closed loops, based at some fixed point, in the lattice.

In applications of the following result to gauge theory, $\mu_{\mathrm{YM}}$ would be the Yang-Mills (Euclidean) quantum gauge field measure (defined heuristically by the formal expression $d \mu_{\mathrm{YM}}=Z^{-1} e^{-S(\omega)}[\mathscr{D} \omega]$, where $S(\omega)$ is the Yang-Mills action functional, $[\mathscr{D} \omega]$ an informal infinite-dimensional 'Lebesgue measure' on $\Omega$, and $Z$ a normalization constant), and the result shows that this measure is determined by the Wilson loop expectation values (**) (and gaugeinvariance). The space $\Omega$ would correspond (heuristically) to the quotient $\mathscr{A} / \mathscr{G}_{m}$, where $\mathscr{G}_{m}$ is the group of all gauge transformations $\phi$ of $P$ which fix $u ; \mathscr{G}_{m}$ acts on $\mathscr{A}$ by $(\phi, \omega) \mapsto \phi^{*} \omega$.

Theorem 4. Let $\left(\Omega, \mathscr{F}, \mu_{\mathrm{YM}}\right)$ be a probability measure space, and suppose that for every closed loop $K \in \mathscr{K}$ there is given a $G$-valued random variable $\Omega \rightarrow G: \omega \mapsto g(C ; \omega) \in G$. Here $G$ is any compact group to which Theorem 1 applies. Assume that $\mathscr{F}$ is the $\sigma$-algebra generated by the functions $\omega \mapsto g(C ; \omega)$ as $C$ runs over $\mathscr{H}$. Assume also that for every positive integer $m, C_{1}, \ldots, C_{m} \in \mathscr{K}$, and every $x \in G$, the $G^{m}$-valued random variable $\omega \mapsto\left(x g\left(C_{1} ; \omega\right) x^{-1}, \ldots, x g\left(C_{m} ; \omega\right) x^{-1}\right)$ has the same distribution (under $\left.\mu_{\mathrm{YM}}\right)$ as $\omega \mapsto\left(g\left(C_{1} ; \omega\right), \ldots, g\left(C_{m} ; \omega\right)\right)$. Now suppose $\mu_{\mathrm{YM}}^{\prime}$ is a probability measure on $(\Omega, \mathscr{F})$ such that all the preceding hypotheses are valid with $\mu_{\mathrm{YM}}$ replaced by $\mu_{\mathrm{YM}}^{\prime}$. Assume finally that

$$
\begin{aligned}
\int_{\Omega} \chi_{1}\left(g\left(C_{1} ; \omega\right)\right) \cdots \chi_{r}\left(g\left(C_{r} ; \omega\right)\right) d \mu_{\mathrm{YM}}(\omega) \\
=\int_{\Omega} \chi_{1}\left(g\left(C_{1} ; \omega\right)\right) \cdots \chi_{r}\left(g\left(C_{r} ; \omega\right)\right) d \mu_{\mathrm{YM}}^{\prime}(\omega)
\end{aligned}
$$

for every positive integer $r$, every collection of curves $C_{1}, \ldots, C_{r} \in \mathscr{K}$, and every choice of characters $\chi_{1}, \ldots, \chi_{r}$ of $G$.

Then $\mu_{\mathrm{YM}}=\mu_{\mathrm{YM}}^{\prime}$.

Proof. For positive integer $j$ denote by $\mathscr{A}_{j}$ the space of all continuous functions $f: G^{j} \rightarrow C$ which satisfy $f\left(g x_{1} g^{-1}, \ldots, g x_{j} g^{-1}\right)=f\left(x_{1}, \ldots, x_{j}\right)$ for every $g, x_{1}, \ldots, x_{j} \in G$. Then $\mathscr{A}_{j}$ is an algebra under pointwise addition and 
multiplication. Let $\mathscr{B}_{j}$ be the subalgebra of $\mathscr{A}_{j}$ generated by functions of the form $\left(g_{1}, \ldots, g_{j}\right) \mapsto \chi\left(g_{i_{1}} \cdots g_{i_{k}}\right)$, where $\chi$ ranges over all characters of $G$, $k$ over all positive integers, and $i_{1}, \ldots, i_{k}$ over $\{1, \ldots, j\}$.

By Theorem 2, if $x, y \in G^{j}$ are such that $f(x)=f(y)$ for every $f \in \mathscr{B}_{j}$ then $f(x)=f(y)$ for every $f \in \mathscr{A}_{j}$. By going down to the quotient $G^{j} / G$ using the action of $G$ on $G^{j}$ given by $\left(g,\left(x_{1}, \ldots, x_{j}\right)\right) \mapsto\left(g x_{1} g^{-1}, \ldots, g x_{j} g^{-1}\right)$, it follows from the Stone-Weierstrass Theorem that $\mathscr{B}_{j}$ is dense in the supremum norm in $\mathscr{A}_{j}$. That is, if $f \in \mathscr{A}_{j}$ then there is a sequence $\left(f_{k}\right)$ in $\mathscr{B}_{j}$ such that $\sup _{x \in G^{j}}\left|f(x)-f_{k}(x)\right| \rightarrow 0$ as $k \rightarrow \infty$. Therefore, for every positive integer $j$, every $f \in \mathscr{A}_{j}$, and every $C_{1}, \ldots, C_{j} \in \mathscr{K}$,

$$
\begin{aligned}
& \int_{\Omega} f\left(g\left(C_{1} ; \omega\right), \ldots, g\left(C_{j} ; \omega\right)\right) d \mu_{\mathrm{YM}}(\omega) \\
& \quad=\int_{\Omega} f\left(g\left(C_{1} ; \omega\right), \ldots, g\left(C_{j} ; \omega\right)\right) d \mu_{\mathrm{YM}}^{\prime}(\omega) .
\end{aligned}
$$

Here we have used the hypothesis $(* *)$ and the fact that $\mathscr{K}$ is closed under composition of loops. From the invariance properties of $\mu_{\mathrm{YM}}$ and $\mu_{\mathrm{YM}}^{\prime}$ (to be more precise, invariance properties of the distributions of $\left(g\left(C_{1} ;-\right), \ldots\right.$, $\left.g\left(C_{j} ;-\right)\right)$ under $\mu_{\mathrm{YM}}$ and $\left.\mu_{\mathrm{YM}}^{\prime}\right)$ in the hypotheses, it follows, by using an averaging argument, that $(* * *)$ holds for every continuous function $f: G^{j} \rightarrow C$. Therefore, the distribution on $G^{j}$ of $\omega \mapsto\left(g\left(C_{1} ; \omega\right), \ldots, g\left(C_{j} ; \omega\right)\right)$ under $\mu_{\mathrm{YM}}$ and under $\mu_{\mathrm{YM}}^{\prime}$ is the same. Since the random variables $\omega \mapsto g(C ; \omega)$, as $C$ varies over $\mathscr{K}$, generate the $\sigma$-algebra $\mathscr{F}$, we conclude that $\mu_{\mathrm{YM}}=$ $\mu_{\mathrm{YM}}^{\prime}$.

Remarks. After completion of most of this work I became aware of the work [Du] where, among other results of related interest, Theorem 2 is proved for the case of unitary and special unitary groups by a different method. The work [Pr] also contains results closely connected with Theorem 2.

\section{ACKNOWLEDGMENT}

I thank Frederic Bien, Jose Vargas, and Mark Davidson for many useful discussions. The counterexamples in $\S 2$ arose from an example I learned from F. Bien. I am grateful to Piotr Szafranski for helping me locate reference [Du].

\section{REFERENCES}

[Bu1] W. Burnside, Theory of groups of finite order, 2nd ed., Dover, New York, 1911.

[Bu2] _ Proc. London Math. Soc. (2) 11 (1913), 40.

[Dr] B. K. Driver, Classification of bundle connection pairs by parallel translation and lassos, $\mathrm{J}$. Funct. Anal. 83 (1989), 185-231.

[Du] B. Durhuus, On the structure of gauge invariant classical observables in lattice gauge theories, Lett. Math. Phys. 4 (1980), 515-522.

[Fr] J. Frohlich, On the construction of quantized gauge fields, Field Theoretical Methods in Particle Physics (W. Ruhl, ed.), Plenum Press, New York, 1980.

[Gi] R. Giles, Reconstruction of gauge potentials from Wilson loops, Phys. Rev. D 24 (1981), 216-2168.

[GKS] C. King, L. Gross, and A. Sengupta, Two dimensional Yang-Mills theory via stochastic differential equations, Ann. Physics 194 (1989), 65-112. 
[KN] S. Kobayashi and K. Nomizu, Foundations of differential geometry, Vol. I, Wiley, New York, 1963.

[Ko] S. Kobayashi, La connexion des varietes fibrees, C. R. Acad. Sci. Paris Sér. I Math. 238 (1954), 443-444.

[Pr] C. Procesi, The invariant theory of $n \times n$ matrices, Adv. Math. 19 (1976), 306-381.

[Sei] E. Seiler, Gauge theories as a problem of constructive quantum field theory and statistical mechanics, Springer-Verlag, New York, 1982.

[Va] V. S. Varadarajan, Geometry of quantum theory, Springer-Verlag, New York, 1985.

[We] H. Weyl, Group theory and quantum mechanics, Dover, New York, 1950.

Department of Mathematics, Louisiana State University, Baton Rouge, Louisiana 70803

E-mail address: sengupta@marais.math.lsu.edu 